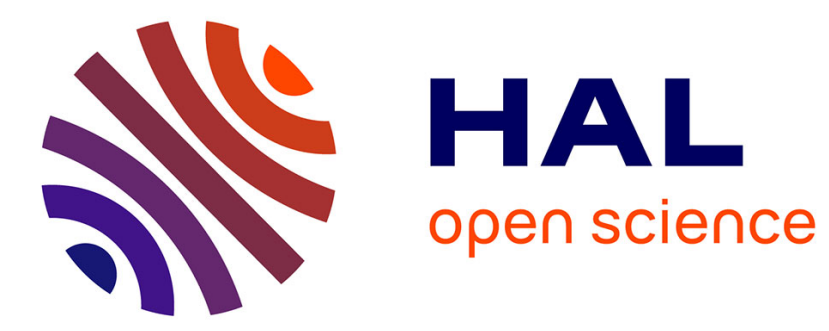

\title{
Path-connectedness of tensor ranks
}

Yang Qi, Pierre Comon, Lek-Heng Lim, Ke Ye

\section{To cite this version:}

Yang Qi, Pierre Comon, Lek-Heng Lim, Ke Ye. Path-connectedness of tensor ranks. EUSIPCO 2019 - 27th European Signal Processing Conference, Sep 2019, A Coruna, Spain. 10.23919/EUSIPCO.2019.8903027 . hal-02121152

\section{HAL Id: hal-02121152 \\ https://hal.science/hal-02121152}

Submitted on 6 May 2019

HAL is a multi-disciplinary open access archive for the deposit and dissemination of scientific research documents, whether they are published or not. The documents may come from teaching and research institutions in France or abroad, or from public or private research centers.
L'archive ouverte pluridisciplinaire HAL, est destinée au dépôt et à la diffusion de documents scientifiques de niveau recherche, publiés ou non, émanant des établissements d'enseignement et de recherche français ou étrangers, des laboratoires publics ou privés. 


\section{Path-connectedness of tensor ranks}

\author{
Yang Qi \\ Department of Mathematics \\ University of Chicago \\ Chicago, USA \\ yangqi@math.uchicago.edu
}

\author{
Pierre Comon \\ Univ. Grenoble Alpes, CNRS \\ Grenoble INP, GIPSA-Lab \\ St Martin d'Hères Cedex, France \\ pierre.comon@gipsa-lab.fr
}

\author{
Lek-Heng Lim \\ Department of Statistics \\ University of Chicago \\ Chicago, USA \\ lekheng@galton.uchicago.edu
}

\section{$\mathrm{Ke} \mathrm{Ye}$}

Academy of Mathematics and Systems Science

Chinese Academy of Sciences

Beijing, China

keyk@amss.ac.cn

\begin{abstract}
Computations of low-rank approximations of tensors often involve path-following optimization algorithms. In such cases, a correct solution may only be found if there exists a continuous path connecting the initial point to a desired solution. We will investigate the existence of such a path in sets of low-rank tensors for various notions of ranks, including tensor rank, border rank, multilinear rank, and their counterparts for symmetric tensors.

Index Terms-Tensor rank, symmetric rank, border rank, multilinear rank, symmetric multilinear rank, pathconnectedness
\end{abstract}

\section{INTRODUCTION}

Given a $d$-tensor $A \in \mathbb{F}^{n_{1} \times \cdots \times n_{d}}:=\mathbb{F}^{n_{1}} \otimes \cdots \otimes \mathbb{F}^{n_{d}}$ over $\mathbb{F}=\mathbb{R}$ or $\mathbb{C}$, its tensor rank $\operatorname{rk}(A)$ is the smallest integer $r$ such that

$$
A=\sum_{i=1}^{r} v_{1, i} \otimes \cdots \otimes v_{d, i}
$$

for some vectors $v_{j, i} \in \mathbb{F}^{n_{j}}, j=1, \ldots, d, i=1, \ldots, r$. A flattening $b_{i}$ maps $A$ to a matrix by 'forgetting' the tensor product structure,

$$
b_{i}: \mathbb{F}^{n_{1} \times \cdots \times n_{d}} \rightarrow \mathbb{F}^{n_{i}} \otimes \mathbb{F}^{n_{1} \cdots n_{i-1} n_{i+1} \cdots n_{d}} .
$$

In other words, $b_{i}(A)$ is the $n_{i} \times n_{1} \cdots n_{i-1} n_{i+1} \cdots n_{d}$ matrix given by

$$
\left[\begin{array}{ccc}
A_{1 \cdots 1} & \cdots & A_{n_{1} \cdots n_{i-1} 1 n_{i+1} \cdots n_{d}} \\
\vdots & \ddots & \vdots \\
A_{1 \cdots 1 n_{i} 1 \cdots 1} & \cdots & A_{n_{1} \cdots n_{d}}
\end{array}\right]
$$

for $i=1, \ldots, d$. The multilinear rank $\mu \mathrm{rk}(A)$ is defined to be

$$
\mu \operatorname{rk}(A)=\left(\operatorname{rk}\left(b_{1}(A)\right), \ldots, \operatorname{rk}\left(b_{d}(A)\right)\right) .
$$

LHL and YQ are supported by DARPA D15AP00109 and NSF IIS 1546413. In addition, LHL's work is also supported by a DARPA Director's Fellowship and the Eckhardt Faculty Fund. KY's work is supported by NSFC no. 11688101 , NSFC no. 11801548 , National Key R\&D Program of China Grant no. 2018YFA0306702 and the Thousand Talents Program.
Equivalently, $\mu \mathrm{rk}(A)$ is the smallest ${ }^{1}$ tuple $\left(r_{1}, \ldots, r_{d}\right)$ such that $A$ belongs to $\mathbb{F}^{r_{1} \times \cdots \times r_{d}}$ after a change-ofbases. When $d=2$, the multilinear rank $\left(r_{1}, r_{2}\right)$ of a matrix $A$ has $r_{1}$ and $r_{2}$ given by the row and column ranks of $A$ respectively.

For a symmetric $d$-tensor $A \in \mathrm{S}^{d}\left(\mathbb{F}^{n}\right)$, the symmetric rank $\operatorname{rks}_{\mathrm{S}}(A)$ is the smallest integer $r$ such that

$$
A=\sum_{i=1}^{r} v_{i}^{\otimes d}
$$

for some vectors $v_{i} \in \mathbb{F}^{n}, i=1, \ldots, r$. The symmetric multilinear rank $\mu \mathrm{rk}_{\mathrm{S}}(A)$ is the minimum integer $r$ such that $A$ belongs to $\mathrm{S}^{d}\left(\mathbb{F}^{r}\right)$ after a change-of-basis. For a symmetric tensor $A \in \mathrm{S}^{d}\left(\mathbb{F}^{n+1}\right)$, its $\operatorname{rank} \operatorname{rk}(A)$ and symmetric rank $\operatorname{rks}_{\mathrm{S}}(A)$ may be different [18]. On the other hand, we always have $\mu \operatorname{rk}(A)=(r, \ldots, r)$ where $r=\mu \mathrm{rk}_{\mathrm{S}}(A)$.

We note that symmetric $d$-tensors are in bijective correspondence with homogeneous degree- $d$ polynomials; e.g., for $d=3$, any $A=\left(a_{i j k}\right) \in \mathrm{S}^{3}\left(\mathbb{C}^{n}\right)$ corresponds to $\sum_{i, j, k=1}^{n} a_{i j k} x_{i} x_{j} x_{k} \in \mathbb{C}\left[x_{1}, \ldots, x_{n}\right]$. So our results on symmetric tensors apply verbatim to homogeneous polynomials.

When $d \geq 3$ and $r>1$, the set of tensors of rank at most $r$, i.e., $\left\{A \in \mathbb{F}^{n_{1} \times \cdots \times n_{d}} \mid \operatorname{rk}(A) \leq r\right\}$, and the set of symmetric tensors of symmetric rank at most $r$, i.e., $\left\{A \in \mathrm{S}^{d}\left(\mathbb{F}^{n}\right) \mid \operatorname{rks}_{\mathrm{S}}(A) \leq r\right\}$, are not necessarily closed in the Euclidean topology [10], [14], which necessitates the notions of border rank brk $(A)$ : this is the smallest integer $r$ such that $A$ is a limit of rank$r$ tensors; likewise, the symmetric border rank brks $(A)$ of a symmetric tensor $A$ is the smallest integer $r$ such that $A$ is a limit of symmetric rank- $r$ tensors.

Determining best low-rank approximations of a tensor (when they exist) with respect to one of the aforementioned ranks is an important problem in applications.

\footnotetext{
${ }^{1}$ Here 'smallest' is with respect to the partial order on $\mathbb{N}^{d}$ defined by $\left(t_{1}, \ldots, t_{d}\right) \leq\left(s_{1}, \ldots, s_{d}\right)$ if $t_{j} \leq s_{j}$ for all $j=1, \ldots, d$.
} 
Riemannian manifold optimization techniques [2], [11] have often been used, particularly in the best multilinear rank approximation problem [12], [17]. In particular, the authors of [13] proposed to find a best approximation of a given tensor in the set of fixed multilinear rank tensors, i.e., optimize over the set

$X_{r_{1}, \ldots, r_{d}}:=\left\{A \in \mathbb{F}^{n_{1} \times \cdots \times n_{d}} \mid \mu \operatorname{rk}(A)=\left(r_{1}, \ldots, r_{d}\right)\right\}$,

instead of the set

$\bar{X}_{r_{1}, \ldots, r_{d}}=\left\{A \in \mathbb{F}^{n_{1} \times \cdots \times n_{d}} \mid \mu \operatorname{rk}(A) \leq\left(r_{1}, \ldots, r_{d}\right)\right\}$,

the reason being that $X_{r_{1}, \ldots, r_{d}}$ is a smooth Riemannian manifold [20] whereas $\bar{X}_{r_{1}, \ldots, r_{d}}$ has singular points. However, as these Riemannian manifold optimization techniques are path-following algorithms, we need to know if $X_{r_{1}, \ldots, r_{d}}$ is path-connected or not. If not, a path-following algorithm starting from one connected component can never reach optimizers located in other components.

In this article, we summarize our path-connectedness results for the following sets:

(1) $\left\{A \in \mathbb{F}^{n_{1} \times \cdots \times n_{d}} \mid \operatorname{rk}(A)=r\right\}$,

(2) $\left\{A \in \mathrm{S}^{d}\left(\mathbb{F}^{n}\right) \mid \operatorname{rk}_{\mathrm{S}}(A)=r\right\}$,

(3) $\left\{A \in \mathbb{F}^{n_{1} \times \cdots \times n_{d}} \mid \operatorname{brk}(A)=r\right\}$,

(4) $\left\{A \in \mathrm{S}^{d}\left(\mathbb{F}^{n}\right) \mid \operatorname{brk}_{\mathrm{S}}(A)=r\right\}$,

(5) $\left\{A \in \mathbb{F}^{n_{1} \times \cdots \times n_{d}} \mid \mu \operatorname{rk}(A)=\left(r_{1}, \ldots, r_{d}\right)\right\}$,

(6) $\left\{A \in \mathrm{S}^{d}\left(\mathbb{F}^{n}\right) \mid \mu \mathrm{rk}_{\mathrm{S}}(A)=r\right\}$,

for $d \geq 3$ over both $\mathbb{F}=\mathbb{R}$ and $\mathbb{C}$. These sets are pathconnected over $\mathbb{C}$ if $r$ is strictly less than the generic rank, but the situation is more subtle over $\mathbb{R}$. Roughly speaking, (1), (3), (5) are path-connected over $\mathbb{R}$ when $r$ is no more than the generic rank; with the requirement that the order of the tensor $d$ is odd, so are (2), (4), (6).

This article contains a digest of selected results in [9] that provide theoretical guarantees for Riemannian optimization algorithms used in low-rank tensor approximations. For complete proofs and additional topological properties (e.g., fundamental groups and higher homotopy groups), we refer readers to [9].

\section{II. $X$-RANK}

As we would like to study path-connectedness, a topological property, it is natural and convenient to use geometric language. In the next section, we will restate the various notions of ranks in terms of $X$-ranks, where $X$ is a complex irreducible projective variety. We refer readers to [14] for more information.

\section{A. $X$-rank and $X$-border rank}

To employ the framework of classical algebraic geometry, we will work in projective spaces instead of affine spaces, where we have more powerful tools and nicer properties of varieties and morphisms between varieties. The projective space $\mathbb{C P}^{n}$ is the set of lines in $\mathbb{C}^{n+1}$ passing through 0 . For a nonzero vector $v \in \mathbb{C}^{n+1}$, a point $[v] \in \mathbb{C P}^{n}$ represents the line $\{\lambda v \mid \lambda \in \mathbb{C}\}$. This definition gives rise to a quotient map

$$
\pi: \mathbb{C}^{n+1} \backslash\{0\} \rightarrow \mathbb{C P}^{n}, \quad v \mapsto[v] .
$$

As a quotient space, $\mathbb{C P}^{n}$ has a natural quotient topology induced by the Euclidean topology on $\mathbb{C}^{n+1}$, and this topology on $\mathbb{C P}^{n}$ is also called the Euclidean topology, which also makes $\mathbb{C P}^{n}$ a smooth complex compact manifold. For any subset $X \subseteq \mathbb{C P}^{n}$, the set $\widehat{X}:=$ $\pi^{-1}(X) \cup\{0\}$ is called the affine cone over $X$.

A complex projective variety in $\mathbb{C P}^{n}$ is defined to be the zero locus of finitely many homogeneous polynomials in $n+1$ variables. Given a complex projective variety $X \subseteq \mathbb{C P}^{n}$, a subset $Y \subseteq X$ is called a subvariety of $X$ if $Y$ is itself a projective variety in $\mathbb{C P}^{n}$, i.e., the zero locus of finitely many homogeneous polynomials. As a subset of $\mathbb{C P}^{n}$, any projective variety $X \subseteq \mathbb{C P}^{n}$ inherits the Euclidean topology from $\mathbb{C P}^{n}$. We say $X$ is smooth if it is a smooth manifold in the Euclidean topology. Our discussions up to this point also apply verbatim to $\mathbb{R P}^{n}$, i.e., with $\mathbb{R}$ in place of $\mathbb{C}$.

Given a projective variety $X$, the union of two subvarieties of $X$ is a projective subvariety, and the intersection of a family of subvarieties of $X$ is also a projective subvariety. This property gives a new topology on $X$ where closed subsets of $X$ are exactly projective subvarieties of $X$. This new topology is called the Zariski topology on $X$.

A projective variety $X$ is called nondegenerate if $X$ is not contained in a hyperplane, and $X$ is called irreducible if it is not a union of two nonempty proper projective subvarieties. It is known that any projective variety can be decomposed as a union of finitely many irreducible projective subvarieties.

To connect projective varieties with tensors, we look at some examples that we will use in the next sections. The Segre map is

$$
\begin{aligned}
\text { Seg: } \mathbb{C P}^{n_{1}-1} \times \cdots \times \mathbb{C P}^{n_{d}-1} & \rightarrow \mathbb{C P}^{n_{1} \cdots n_{d}-1}, \\
\left(\left[v_{1}\right], \ldots,\left[v_{d}\right]\right) & \mapsto\left[v_{1} \otimes \cdots \otimes v_{d}\right] .
\end{aligned}
$$

Its image $\operatorname{Seg}\left(\mathbb{C P}^{n_{1}-1} \times \cdots \times \mathbb{C P}^{n_{d}-1}\right)$ is an irreducible smooth projective variety and is called the Segre variety. If we let $X=\operatorname{Seg}\left(\mathbb{C P}^{n_{1}-1} \times \cdots \times \mathbb{C P}^{n_{d}-1}\right)$, then $\widehat{X} \backslash\{0\}$ is the set of rank-one $d$-tensors. Likewise, the Veronese map is

$$
\nu_{d}: \mathbb{C P}^{n-1} \rightarrow \mathbb{P S}^{d}\left(\mathbb{C}^{n}\right), \quad[v] \mapsto\left[v^{\otimes d}\right] .
$$

Its image $\nu_{d}\left(\mathbb{C P}^{n-1}\right)$ is an irreducible smooth projective variety and is called the Veronese variety. If we let $X=$ $\nu_{d}\left(\mathbb{C} \mathbb{P}^{n-1}\right)$, then $\widehat{X} \backslash\{0\}$ is the set of symmetric rankone $d$-tensors. 
The ideal of a projective variety $X \subseteq \mathbb{C P}^{n}$ is the set of those homogeneous polynomials in $\mathbb{C}\left[x_{1}, \ldots, x_{n+1}\right]$ that vanish on $X$. If the ideal of $X$ is generated by homogeneous polynomials with real coefficients, then the set of real points $X(\mathbb{R})$ of $X$ is defined to be the zero locus of these polynomials in $\mathbb{R} \mathbb{P}^{n}$. Although $X$ and $X(\mathbb{R})$ are defined by the same polynomials, their properties can be vastly different. For example, let $X$ be the smooth conic curve in $\mathbb{C P}^{2}$ defined by $x^{2}+y^{2}+z^{2}=0$, which is isomorphic to $\mathbb{C P}^{1}$. On the other hand, it is evident that $X(\mathbb{R})$, i.e., the zero locus of $x^{2}+y^{2}+z^{2}=0$ in $\mathbb{R P}^{2}$, is empty. To avoid such phenomena, in this article we will require that $X(\mathbb{R})$ be Zariski dense in $X$, which is equivalent to requiring that $X$ has a smooth real point [5].

If $X$ is nondegenerate in $\mathbb{C P}^{n}$, then any vector $v \in$ $\mathbb{C}^{n+1}$ can be written as a finite sum of points in $\widehat{X}$. For instance, as $X=\operatorname{Seg}\left(\mathbb{C P}^{n_{1}-1} \times \cdots \times \mathbb{C P}^{n_{d}-1}\right)$ is nondegenerate, any tensor in $\mathbb{C}^{n_{1} \times \cdots \times n_{d}}$ is a finite sum of rank-one tensors, i.e., points in $\widehat{X}$ (see Section II-B). More generally, define the sum-of-r-terms map $s_{r}$ by

$$
s_{r}: \widehat{X}^{r} \rightarrow \mathbb{C}^{n+1}, \quad\left(x_{1}, \ldots, x_{r}\right) \mapsto x_{1}+\cdots+x_{r},
$$

and let $s_{r}(X)$ be its image. The Euclidean closure of $s_{r}(X)$ is in fact Zariski closed, which means that the Euclidean closure of $s_{r}(X)$ can be realized as the affine cone of a certain projective variety in $\mathbb{C P}^{n}$, i.e., the zero locus of finitely many homogeneous polynomials in $n+1$ variables. This variety is called the $r$ th secant variety of $X$ and denoted by $\sigma_{r}(X)$. If $\bar{s}_{r}(X)$ denotes the Euclidean closure of $s_{r}(X)$ and $\widehat{\sigma}_{r}(X)$ the affine cone of $\sigma_{r}(X)$, then

$$
\bar{s}_{r}(X)=\widehat{\sigma}_{r}(X) .
$$

The image of $\widehat{X}(\mathbb{R})^{r}$ under $s_{r}$, denoted by $s_{r}(X(\mathbb{R}))$, is semialgebraic. However its Euclidean closure in $\mathbb{R}^{n+1}$ is not necessarily Zariski closed. The Zariski closure of $s_{r}(\widehat{X}(\mathbb{R}))$ over $\mathbb{R}$ is the affine cone of some real projective variety in $\mathbb{R P}^{n}$. We will denote this real projective subvariety by $\sigma_{r}(X(\mathbb{R}))$ and call it the $r$ th secant variety of $X(\mathbb{R})$. If $X(\mathbb{R})$ is Zariski dense in $X$, then by [7], [16], the $r$ th secant variety of $X(\mathbb{R})$ is the set of real points of the $r$ th secant variety of $X$, i.e.,

$$
\sigma_{r}(X(\mathbb{R}))=\left(\sigma_{r}(X)\right)(\mathbb{R}),
$$

which is Zariski dense in $\sigma_{r}(X)$.

In this article, we deal only with irreducible nondegenerate complex projective varieties $X \subseteq \mathbb{C P}^{n}$ defined by real homogeneous polynomials and whose real points $X(\mathbb{R})$ are Zariski dense. Under this assumption, for any $p \in \mathbb{C}^{n+1}$, the $X$-rank of $p$, denoted $\operatorname{rk}_{X}(p)$, is the minimum integer $r$ such that $p \in s_{r}(X) \backslash s_{r-1}(X)$. The $X$-border rank of $p$, denoted $\operatorname{brk}_{X}(p)$, is the minimum $r$ such that $p \in \bar{s}_{r}(X) \backslash \bar{s}_{r-1}(X)$. Similarly, we define the $X(\mathbb{R})$-rank of $p \in \mathbb{R}^{n+1}$ to be the minimum $r$ such that $p \in s_{r}(X(\mathbb{R})) \backslash s_{r-1}(X(\mathbb{R}))$, and the $X(\mathbb{R})$-border rank of $p$ to be the minimum $r$ such that $p \in \bar{s}_{r}(X(\mathbb{R})) \backslash \bar{s}_{r-1}(X(\mathbb{R}))$. In general, it may happen that

$$
\operatorname{rk}_{X}(p) \neq \operatorname{rk}_{X(\mathbb{R})}(p) \quad \text { or } \operatorname{brk}_{X}(p) \neq \operatorname{brk}_{X(\mathbb{R})}(p),
$$

which requires us to study the real and complex cases separately.

For a fixed $X \subseteq \mathbb{C P}^{n}$, there is a unique integer $r_{g}(X)$, the generic $X$-rank, such that $s_{r_{g}(X)}(X) \backslash s_{r_{g}(X)-1}(X)$ contains a nonempty open subset of $\mathbb{C}^{n+1}$ in the Euclidean topology. We say that $r$ is a typical $X(\mathbb{R})$-rank if $s_{r}(X(\mathbb{R})) \backslash s_{r-1}(X(\mathbb{R}))$ contains a nonempty Euclidean open subset of $\mathbb{R}^{n+1}$. It turns out that $r_{g}(X)$ always equals the smallest typical $X(\mathbb{R})$-rank [5].

\section{B. Tensor rank and symmetric rank}

We now view tensor rank and symmetric rank in Section I under the light of $X$-ranks introduced in Section II-A. Let $X=\operatorname{Seg}\left(\mathbb{C P}^{n_{1}-1} \times \cdots \times \mathbb{C P}^{n_{d}-1}\right)$. Then $X(\mathbb{R})=\operatorname{Seg}\left(\mathbb{R P}^{n_{1}-1} \times \cdots \times \mathbb{R P}^{n_{d}-1}\right)$. For any tensor $A \in \mathbb{C}^{n_{1} \times \cdots \times n_{d}}$, we have

$$
\operatorname{rk}(A)=\operatorname{rk}_{X}(A) \quad \text { and } \operatorname{brk}(A)=\operatorname{brk}_{X}(A) .
$$

If $A \in \mathbb{R}^{n_{1} \times \cdots \times n_{d}}$, then

$$
\operatorname{rk}_{\mathbb{R}}(A)=\operatorname{rk}_{X(\mathbb{R})}(A) \quad \text { and } \quad \operatorname{brk}_{\mathbb{R}}(A)=\operatorname{brk}_{X(\mathbb{R})}(A) .
$$

Now let $X=\nu_{d}\left(\mathbb{C P}^{n}\right)$. Then $X(\mathbb{R})=\nu_{d}\left(\mathbb{R P}^{n}\right)$. For any symmetric tensor $A \in \mathrm{S}^{d}\left(\mathbb{C}^{n+1}\right)$, we have

$$
\operatorname{rk}_{\mathrm{S}}(A)=\operatorname{rk}_{X}(A) \quad \text { and } \operatorname{brk}_{\mathrm{S}}(A)=\operatorname{brk}_{X}(A) .
$$

If $A \in \mathrm{S}^{d}\left(\mathbb{R}^{n+1}\right)$, then

$$
\operatorname{rk}_{\mathrm{S}}(A)=\operatorname{rk}_{X(\mathbb{R})}(A) \quad \text { and } \operatorname{brks}_{\mathrm{S}}(A)=\operatorname{brk}_{X(\mathbb{R})}(A) .
$$

The use of $X$-rank will permit us to address the cases (1)-(4) in an unified manner. As a side remark, (5) and (6) are smooth manifolds, but (1)-(4) are not necessarily manifolds.

\section{PATH-CONNECTEDNESS OF COMPLEX RANKS}

Now that we have formulated ranks and border ranks geometrically, the path-connectedness of border $X$-rank over $\mathbb{C}$ becomes clear by the following facts:

(a) $\sigma_{r-1}(X) \subsetneq \sigma_{r}(X)$ whenever $r \leq r_{g}(X)$ [3];

(b) $\sigma_{r}(X) \backslash \sigma_{r-1}(X)$ is path-connected if $\sigma_{r-1}(X) \neq$ $\sigma_{r}(X)$ [15, Corollary 4.16].

We may then deduce a path-connectedness result for $X$ border rank- $r$ points.

Theorem 1: If $r \leq r_{g}(X)$, then the sets $\left\{p \in \mathbb{C}^{n+1} \mid\right.$ $\left.\operatorname{brk}_{X}(p)=r\right\}$ and $\left\{p \in \mathbb{C}^{n+1} \mid \operatorname{rk}_{X}(p)=r\right\}$ are pathconnected. 
The path-connectedness of rank, border rank, symmetric rank, and symmetric border rank over $\mathbb{C}$ are consequences of Theorem 1.

Corollary 1: If $r$ is not more than the generic tensor rank, then

$$
\begin{gathered}
\left\{A \in \mathbb{C}^{n_{1} \times \cdots \times n_{d}} \mid \operatorname{brk}(A)=r\right\}, \\
\left\{A \in \mathbb{C}^{n_{1} \times \cdots \times n_{d}} \mid \operatorname{rk}(A)=r\right\}
\end{gathered}
$$

are both path-connected.

Corollary 2: If $r$ is not more than the generic symmetric rank, then

$$
\begin{gathered}
\left\{A \in \mathrm{S}^{d}\left(\mathbb{C}^{n+1}\right) \mid \operatorname{brk}_{\mathrm{S}}(A)=r\right\}, \\
\left\{A \in \mathrm{S}^{d}\left(\mathbb{C}^{n+1}\right) \mid \operatorname{rks}_{\mathrm{S}}(A)=r\right\}
\end{gathered}
$$

are both path-connected.

\section{PATH-CONNECTEDNESS OF REAL RANKS}

The case of real tensors is more subtle and difficult than complex tensors. We recall from Section II-B that if $X=\nu_{d}\left(\mathbb{C P}^{n}\right)$, then we have $X(\mathbb{R})=\nu_{d}\left(\mathbb{R} \mathbb{P}^{n}\right)$. By Terracini Lemma [19] and Alexander-Hirschowitz Theorem [4], together with the topological fact that removing a semialgebraic subset of codimension at least two from a manifold does not change its pathconnectedness, we obtain our path-connectedness result for symmetric tensor rank and border rank over $\mathbb{R}$.

Theorem 2: Let $n>1$ and $r<\left(\begin{array}{c}n+d \\ d\end{array}\right) /(n+1)$.

(i) If $d$ is odd, then

$$
\begin{aligned}
& \left\{A \in \mathrm{S}^{d}\left(\mathbb{R}^{n+1}\right) \mid \operatorname{rks}_{\mathrm{S}}(A)=r\right\}, \\
& \left\{A \in \mathrm{S}^{d}\left(\mathbb{R}^{n+1}\right) \mid \operatorname{brks}_{\mathrm{S}}(A)=r\right\}
\end{aligned}
$$

are both path-connected.

(ii) If $d$ is even, then

$$
\begin{aligned}
& \left\{A \in \mathrm{S}^{d}\left(\mathbb{R}^{n+1}\right) \mid \operatorname{rks}_{\mathrm{S}}(A)=r\right\}, \\
& \left\{A \in \mathrm{S}^{d}\left(\mathbb{R}^{n+1}\right) \mid \operatorname{brkS}_{\mathrm{S}}(A)=r\right\}
\end{aligned}
$$

both have $r+1$ path-connected components.

To state our path-connectedness results for tensor rank and border rank over $\mathbb{R}$, we will need to bring in the notion of defectivity: We say $X$ is not $r$-defective if

$$
\operatorname{dim}\left(\sigma_{r}(X)\right)=\min \{r \operatorname{dim} X-1, n\}
$$

and $r$-defective otherwise.

By an argument similar to the one that led us to Theorem 2, and an additional assumption to guarantee nondefectivity of $\operatorname{Seg}\left(\mathbb{C P}^{n_{1}-1} \times \cdots \times \mathbb{C P}^{n_{d}-1}\right)$, we obtain the following:

Theorem 3: Suppose $2 \leq n_{1} \leq \cdots \leq n_{d}$. Let $X=$ $\operatorname{Seg}\left(\mathbb{C P}^{n_{1}-1} \times \cdots \times \mathbb{C P}^{n_{d}-1}\right)$ and $r<r_{g}(X)$. If

$$
\begin{aligned}
\operatorname{codim}_{\mathbb{C}}\left(\sigma_{r-1}(X),\right. & \left.\sigma_{r}(X)\right) \\
& >n_{1}+\cdots+n_{d-1}-d+2,
\end{aligned}
$$

then

$$
\begin{aligned}
& \left\{A \in \mathbb{R}^{n_{1} \times \cdots \times n_{d}} \mid \operatorname{rk}(A)=r\right\}, \\
& \left\{A \in \mathbb{R}^{n_{1} \times \cdots \times n_{d}} \mid \operatorname{brk}(A)=r\right\}
\end{aligned}
$$

are both path-connected.

We state an alternative version of Theorem 3 that requires an explicit assumption on defectivity.

Theorem 4: If $\operatorname{Seg}\left(\mathbb{C P}^{n_{1}-1} \times \cdots \times \mathbb{C P}^{n_{d}-1}\right)$ is not $r$-defective, then the sets

$$
\begin{gathered}
\left\{A \in \mathbb{R}^{n_{1} \times \cdots \times n_{d}} \mid \operatorname{rk}(A)=r\right\}, \\
\left\{A \in \mathbb{R}^{n_{1} \times \cdots \times n_{d}} \mid \operatorname{brk}(A)=r\right\}
\end{gathered}
$$

are both path-connected.

Unlike the case of symmetric tensors [4], there are still cases where the dimension of $\sigma_{r}\left(\operatorname{Seg}\left(\mathbb{C P}^{n_{1}-1} \times \cdots \times\right.\right.$ $\left.\mathbb{C P}^{n_{d}-1}\right)$ ), and thus its defectivity, remains unknown. On the other hand, there has been recent remarkable progress [1], [6], [8] that guarantees that when $n_{d}>3$, all known cases satisfy the condition (6) in Theorem 3.

\section{PATH-CONNECTEDNESS OF MULTILINEAR RANK}

If $\left(r_{1}, \ldots, r_{d}\right)$ is the multilinear rank of some tensor $A \in \mathbb{F}^{n_{1} \times \cdots \times n_{d}}$, then by (2) we have

$$
r_{i}=\operatorname{rk}\left(b_{i}(A)\right) \leq \min \left\{n_{i}, \prod_{j \neq i} r_{j}\right\}, \quad i=1, \ldots, d .
$$

Intuitively, the connectedness of the set of tensors of a fixed multilinear rank, by virtue of its definition in terms of matrix rank, ought to be essentially the same as the connectedness of the set of matrices of a fixed rank. This intuition can be made mathematically rigorous by the so-called Kempf-Weyman desingularization [21], which leads to the following results.

Theorem 5:

(i) The set of multilinear rank- $\left(r_{1}, \ldots, r_{d}\right)$ real tensors

$$
\left\{A \in \mathbb{R}^{n_{1} \times \cdots \times n_{d}} \mid \mu \operatorname{rk}(A)=\left(r_{1}, \ldots, r_{d}\right)\right\}
$$

is path-connected if

$$
r_{i}<\prod_{j \neq i} r_{j} \quad \text { for all } i=1, \ldots, d
$$

or if

$$
r_{i}=\prod_{j \neq i} r_{j}<n_{i} \quad \text { for some } i=1, \ldots, d .
$$

(ii) The set of multilinear rank- $\left(r_{1}, \ldots, r_{d}\right)$ real tensors

$$
\left\{A \in \mathbb{R}^{n_{1} \times \cdots \times n_{d}} \mid \mu \operatorname{rk}(A)=\left(r_{1}, \ldots, r_{d}\right)\right\}
$$

has two connected components if

$$
r_{i}=\prod_{j \neq i} r_{j}=n_{i} \quad \text { for some } i=1, \ldots, d .
$$

Theorem 6: The set of multilinear rank- $\left(r_{1}, \ldots, r_{d}\right)$ complex tensors

$$
\left\{A \in \mathbb{C}^{n_{1} \times \cdots \times n_{d}} \mid \mu \operatorname{rk}(A)=\left(r_{1}, \ldots, r_{d}\right)\right\}
$$


is always path-connected.

For symmetric tensors, we may similarly deduce analogous results in terms of symmetric multilinear rank. There are four separate cases to consider over $\mathbb{R}$ but just one over $\mathbb{C}$.

Theorem 7:

(i) When $r=1$ and $d$ is odd, the set of symmetric multilinear rank-one real tensors

$$
\left\{A \in \mathrm{S}^{d}\left(\mathbb{R}^{n}\right) \mid \mu \operatorname{rk}_{\mathrm{S}}(A)=1\right\}
$$

is a path-connected set.

(ii) When $r=1$ and $d$ is even, the set of symmetric multilinear rank-one real tensors

$$
\left\{A \in \mathrm{S}^{d}\left(\mathbb{R}^{n}\right) \mid \mu \mathrm{rk}_{\mathrm{S}}(A)=1\right\}
$$

has two connected components.

(iii) When $d=2$, the set of symmetric multilinear rank$r$ real tensors

$$
\left\{A \in \mathrm{S}^{d}\left(\mathbb{R}^{n}\right) \mid \mu \mathrm{rk}_{\mathrm{S}}(A)=r\right\}
$$

has $r+1$ connected components.

(iv) When $r \geq 2$ and $d \geq 3$, the set of symmetric multilinear rank- $r$ real tensors

$$
\left\{A \in \mathrm{S}^{d}\left(\mathbb{R}^{n}\right) \mid \mu \mathrm{rk}_{\mathrm{S}}(A)=r\right\}
$$

is a path-connected set.

Theorem 8: The set of symmetric multilinear rank- $r$ complex tensors

$$
\left\{A \in \mathrm{S}^{d}\left(\mathbb{C}^{n}\right) \mid \mu \mathrm{rk}_{\mathrm{S}}(A)=r\right\}
$$

is always path-connected.

\section{BEYOND GENERIC RANK}

The case when rank exceeds generic rank is a notable omission from our list of results in this article. In general, when $r$ is strictly greater than the generic rank, the set of (border) rank- $r$ tensors is not path-connected. However, it is usually difficult to determine the number of connected components, and we are unaware of any technique that applies generally towards this end. We end this article with a special case, studied extensively in [10], where we are able to determine the exact number of connected components.

Proposition 1: The set

$$
\left\{A \in \mathbb{R}^{2 \times 2 \times 2} \mid \operatorname{brk}(A)=3\right\}
$$

has four path-connected components.

\section{REFERENCES}

[1] H. Abo, G. Ottaviani, and C. Peterson. Induction for secant varieties of Segre varieties. Trans. Amer. Math. Soc., 361(2):767792, 2009.

[2] P.-A. Absil, R. Mahony, and R. Sepulchre. Optimization algorithms on matrix manifolds. Princeton University Press, Princeton, NJ, 2008. With a foreword by Paul Van Dooren.

[3] B. Ådlandsvik. Joins and higher secant varieties. Math. Scand., 61(2):213-222, 1987

[4] J. Alexander and A. Hirschowitz. Polynomial interpolation in several variables. J. Algebraic Geom., 4(2):201-222, 1995.

[5] G. Blekherman and Z. Teitler. On maximum, typical and generic ranks. Math. Ann., 362(3-4):1021-1031, 2015.

[6] C. Bocci, L. Chiantini, and G. Ottaviani. Refined methods for the identifiability of tensors. Ann. Mat. Pura Appl. (4), 193(6):16911702, 2014.

[7] J. Buczyński and J. Jelisiejew. Finite schemes and secant varieties over arbitrary characteristic. Differential Geom. Appl., 55:13-67, 2017

[8] L. Chiantini, G. Ottaviani, and N. Vannieuwenhoven. An algorithm for generic and low-rank specific identifiability of complex tensors. SIAM J. Matrix Anal. Appl., 35(4):1265-1287, 2014.

[9] P. Comon, L.-H. Lim, Y. Qi, and K. Ye. Topology of tensor ranks. arXiv:1804.08060, 2018.

[10] V. De Silva and L.-H. Lim. Tensor rank and the ill-posedness of the best low-rank approximation problem. SIAM J. Matrix Anal. Appl., 30(3):1084-1127, 2008.

[11] A. Edelman, T. A. Arias, and S. T. Smith. The geometry of algorithms with orthogonality constraints. SIAM J. Matrix Anal. Appl., 20(2):303-353, 1999.

[12] L. Eldén and B. Savas. A Newton-Grassmann method for computing the best multilinear rank- $\left(r_{1}, r_{2}, r_{3}\right)$ approximation of a tensor. SIAM J. Matrix Anal. Appl., 31(2):248-271, 2009.

[13] D. Kressner, M. Steinlechner, and B. Vandereycken. Low-rank tensor completion by Riemannian optimization. BIT, 54(2):447468, 2014.

[14] J. M. Landsberg. Tensors: geometry and applications, volume 128 of Graduate Studies in Mathematics. American Mathematical Society, Providence, RI, 2012.

[15] D. Mumford. Algebraic geometry. I, volume 221 of Grundlehren der Mathematischen Wissenschaften [Fundamental Principles of Mathematical Sciences]. Springer-Verlag, Berlin-New York, 1981. Complex projective varieties, Corrected reprint

[16] Y. Qi, P. Comon, and L.-H. Lim. Semialgebraic geometry of nonnegative tensor rank. SIAM J. Matrix Anal. Appl., 37(4):15561580, 2016.

[17] B. Savas and L.-H. Lim. Quasi-Newton methods on Grassmannians and multilinear approximations of tensors. SIAM J. Sci. Comput., 32(6):3352-3393, 2010.

[18] Y. Shitov. A counterexample to Comon's conjecture. SIAM J. Appl. Algebra Geom., 2(3):428-443, 2018.

[19] A. Terracini. Sulle $v_{k}$ per cui la varietà degli $s_{h}(h+1)$-seganti ha dimensione minore dell'ordinario. Rend. Circ. Mat. Palermo, 31(1):392-396, 1911

[20] A. Uschmajew and B. Vandereycken. The geometry of algorithms using hierarchical tensors. Linear Algebra Appl., 439(1):133166,2013

[21] J. Weyman. Cohomology of vector bundles and syzygies, volume 149 of Cambridge Tracts in Mathematics. Cambridge University Press, Cambridge, 2003. 